\title{
DESIGN METHODS FOR COMPLIANT MECHANISMS USED IN NEW AGE INDUSTRIES - A REVIEW
}

\author{
Pandiyan Arumugam \\ Saveetha University, Department of Mechanical Engineering, India \\ Arun Kumar \\ Saveetha University, Department of Mechanical Engineering, India
}

Compliant mechanisms have made an enormous contribution in various fields. Several methods have being conceived to analyze and design these compliant mechanisms that gain part of their motion from the deflection of flexible members rather than from movable joints only. Traditional rigidbody mechanisms have a number of components to apply their functions. Consequently they face problems such as backlash, wear, and increase in part-count, weight, assembly cost and time, regular maintenance. By reducing these problems will assist in increasing mechanism performance and cost reduction. Recently, many familiar examples of compliant mechanisms have been designed and widely used in various fields such as automotive industry, aerospace industry, MEMS, Medical devices, Robotic arm with minimal impedance due to its own mass \& assistive mechanisms. For adaptive structures, components in transportations, hand-held tools, etc. however, the largest challenge was relative difficulty in analyzing and designing compliant mechanisms. Two approaches studied in the literature of kinematics-based approach and the structural optimization based approach. At present work is being carried out to analyze, the best suitable optimization method for designing a compliant mechanisms used in new age industries.

Keywords: Compliant Mechanisms, Kinematics-based Approach, Structural Optimization Approach

\section{INTRODUCTION}

Mechanism is a mechanical device used to transfer the force, energy and motion. But Compliant Mechanism is a monolithic structure that elastically deforms without any link and joint to produce a desired force or displacement. It is an elastic continuum that is used to transmit or transform force and motion mechanically. Rigidbody mechanisms have rigid links connected at movable joints. Unlike rigid-body mechanism, compliant mechanisms gain some of their mobility from the deflection of flexible members rather than movable joints only.

Stiffness is a measure of load per unit deflection, whereas strength is how much load can be endured before failure. Despite human tendencies, it is possible to make things that are flexible and strong. Nature uses stiff structures where needed (tree trunks, bones, teeth, and claws)but in living organisms, it more often relies on flexibility. Bee wings, bird wings, tree branches, leaf stems, fish, and single-celled organisms are only a few examples of creations that use compliance to their advantage.
The contrast between nature and human design is easily identifiable when humans try to replace one of nature's products. For example, a human heart valve is a compliant one-way valve that is capable of sustaining billions of cycles without failure. However, most current artificial heart valves use a number of assembled stiff parts with pin joints to obtain motion. They also have a comparatively shorter life, because difficulty in blood flow, and often damage blood cells [01].

The familiar examples of compliant mechanisms designed in single-piece that replaced rigidlink mechanisms, Figure 1, shows examples of compliant mechanisms used commonly. Systems with concentrated compliance behave like classic rigid link mechanisms, where kinematic joints are replaced with flexible hinges, and in consequence methods conceived to design rigid body mechanisms can be modified and applied successfully in this case. Design methods for mechanisms with concentrated compliance design had its genesis in the works of Ashok Midha in the 1980s. He developed a tool to classify and design mechanisms with concentrated compliance with his coworkers (Midha et al. (1994). 


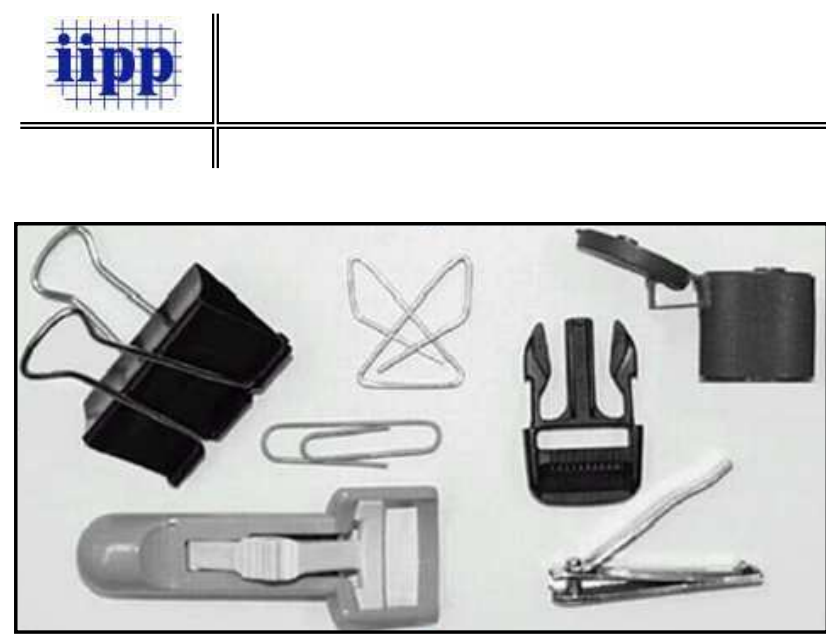

Figure 1: Common compliant devices. A binder clip, paper clip, backpack latch, lid, eyelash curler and nail clippers are shown: [01]

Introduced the pseudo-rigid model concept, where flexible links are modeled as rigid links connected by kinematic joints and torsional springs, and this tool allows design compliant mechanisms with methods conceived to design rigid mechanisms [02].

Methodologies to design mechanisms with distributed compliance appeared in the middle of the 1990s. In this case the mechanism is treated as a continuum flexible structure, and continuum mechanics design methods are used instead of rigid body kinematics. [04]. Pioneered the use of structural optimization applied to the design of compliant mechanisms with distributed compliance, by adapting the homogenization method and using the displacement of one point in the mechanism as the objective function. Alternative structural optimization procedures seek different objective functions, like the minimization of the mechanism's deformation energy [05]. A more recent technique applied to mechanisms with distributed compliance was introduced by Kota et al (1997), where a load path methodology and genetic algorithms are used to design compliant mechanisms with shape-morphing starting from a domain discretized by an exhaustive set of truss or beam elements.

The proposed design method addresses flexibility and stiffness issues of the compliant mechanism simultaneously. It aims to attain an optimal balance between these two conflicting attributes. Such techniques are successful in indirectly controlling the local stress levels by constraining the input displacement [06].

A level-set method for the design of compliant mechanisms, the objective is to design a monolithic compliant mechanism, made by multiple materials. The level set method for designing monolithic compliant mechanism made of multiple materials is an optimization of continuum heterogeneous structures [07].

The design of a compliant mechanism involves three main aspects: (i) topology: the connectivity of material, (ii) size: the cross-sectional area of each segment, and (iii) geometry: the orientations of the connecting segments and locations of the junctions. Several systematic design methods have been developed previously in the Compliant Systems Design Laboratory (CSDL) (8-11). The topology design is treated as a layout optimization problem to search for the optimal material arrangement and connectivity within a given design domain. The design domain must be broken down in such a manner that it can be parameterized and mathematically represented in a systematic fashion. The size and geometry of a given topology can be further optimized to improve functional performance.

\section{Merits \& application of Compliant Mechanisms}

Due to their monolithic (joint less) construction, compliant transmissions offer many inherent benefits including low cost, zero backlash, ease of manufacture, and scalability. It is specified that some mechanisms may be manufactured from an injection-moldable material and be constructed of one piece. Compliant mechanisms also have fewer movable joints such as turning and sliding joints. This results in reduced wear and need for lubrication [01].

New competitive products must meet the growing demands of the market. They must be lightweighted, resource efficient, durable, and stable and have a low noise emission. At the same time, the products must be introduced quickly into the market. For the fulfillment of these demands it is necessary to use the advantages of compliant mechanisms. Compliant mechanisms are applicable in various fields such as for adaptive structures, components in transportations, handheld tools, electronics, robotics, medical, etc for numerous reasons.

For example, Consider the compliant overrunning clutch and its rigid body counterpart shown in Figure 2 (a) and 2 (b). Considerably fewer components are required for the compliant mechanism than for the rigid mechanism. The reduction in part count may reduce manufacturing and assembly time and cost. Compliant overrunning clutch is an example for applications of 
compliant systems in automotive industry, apart from that the car wiper, steering part, gears, brakes and centrifugal clutches are the other examples. The compliant mechanisms and systems are also used in (new age) industries like aerospace, Mechanism amplification for sensors and actuators, Constant force mechanism for microsurgery suturing device, Robotic arm with minimal impedance due to its own mass \& Assistive mechanisms.

(a)

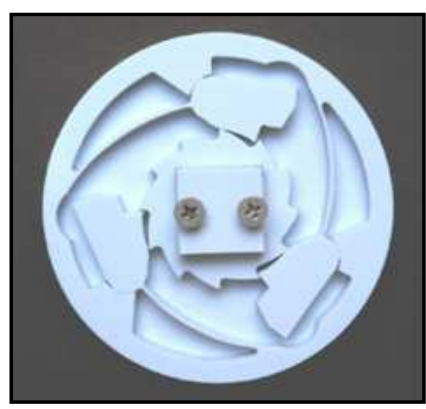

(b)

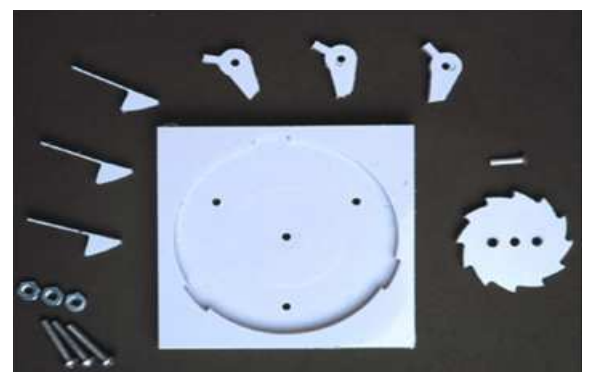

Figure 2: (a) Compliant overrunning clutch (b) Rigidbody counterpart Source: [01]

\section{Challenges \& Demerits of Compliant Mechanisms}

The largest challenge is the relative difficulty in analyzing and designing compliant mechanisms. Since many of the flexible members undergo large deflections, linearized beam equations are no longer valid. Nonlinear equations must be used that account for the geometric nonlinearities caused by large deflections. Because of these difficulties, many compliant mechanisms in the past were designed by trial and error approaches. However, in some applications having energy stored in flexible members is a disadvantage. For example, if a mechanism's function is to transfer energy from the input to an output, not all of the energy is transferred since some is stored in the mechanism. Fatigue analysis is typically a more vital issue for compliant mechanisms than for their rigidbody counterparts. The motion from the deflection of compliant links is also limited by the strength of the deflecting members. Obviously a compliant link cannot produce a continuous rotational mo- tion such as that possible with a pin joint [01].

\section{Fabrication of compliant Mechanisms}

We can simply manufacture a single-piece fully compliant mechanism via injection molding, extrusion and rapid prototyping for medium size devices [12], or using silicon surface micromachining [13] and electroplating techniques [14] for compliant micro-mechanisms. Although a compliant mechanism gives numerous advantages, it is difficult to design and analyze. Much of the current compliant mechanism design, however, must be performed without the aid of a formal synthesis method and is based on designer's intuition and experience [15, 16]. Several trial and error iterations using finite element models are often required to obtain the desired mechanism performance.

\section{SYNTHESIS OF MECHANISMS}

Typically, there are two approaches known in the literature for the systematic syntheses of compliant mechanisms are the kinematics based approach [17] and the structural optimization based approach $[18,19]$.

\section{Kinematics-based approach}

In kinematics approach, compliant segments are illustrated as several rigid links connected together by pin joints and torsional springs are added to resist torsion. The value of spring constants and where to place it to the model are calculated differently depending on types of segments. There are several familiar segments assigned by [17] i.e., small-length flexural pivots, cantilever beam with force at the free end (fixed pinned), fixedguided flexible segment, initially curved cantilever beam and pinned-pinned segment. Different types of segments require different models, see Figure 3 and in Howell discussed briefly how they might be applied to compliant mechanisms.

Although this method is easier to analyze compare to its compliant counterpart, however, mechanism's force-deflection relationships are still difficult to be determined. Typically, there are two approaches introduced to determine that relationship from pseudo rigid- body models. The first method uses conventional Newtonian methods i.e., each links are analyzed to obtain static equilibrium.

Thus, the force system for the entire mechanism is established. On the other hand, principle of virtual work is also can be selected to deter- 
mine force-deflection relationship. The approach views the system entirely and does not include all the reaction forces [01]. Typically, kinematics-based approach is well suited with mechanisms that undergo large, nonlinear deflections. Besides, this approach requires starting with a known rigid-links mechanism.

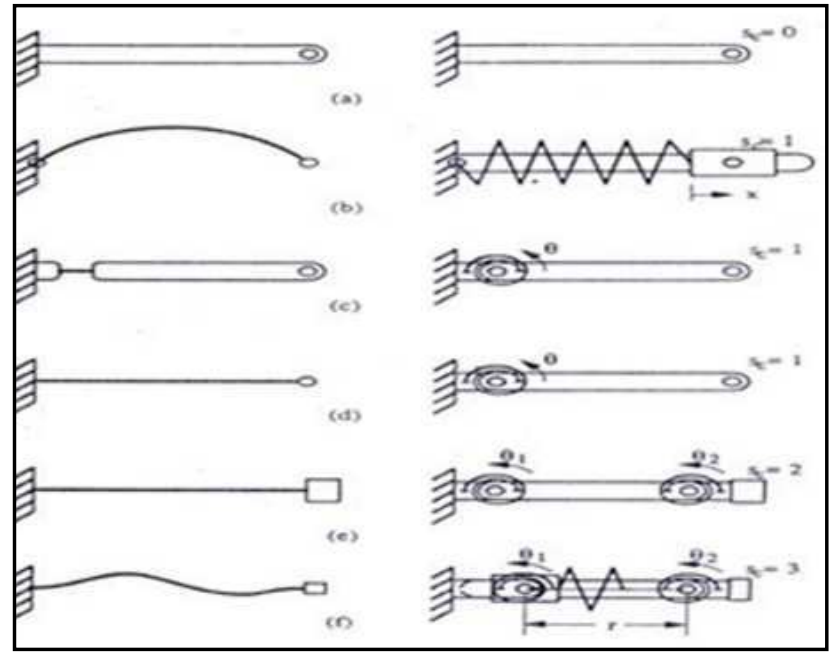

Figure 3: Various flexible segments and their pseudo rigid-body models (Howell L.L. 2003)

\section{Structural optimization based approach}

In this approach it is not required to begin with a known rigid link mechanism. It focuses on the determination of the topology, shape and size of the mechanism [20].

A numerical approach of topology optimization starts with a domain of material to which the external loads and supports are applied [21]. The objective function is often the compliance, that is, the flexibility of the structure under the given loads, subject to a volume constraint. In general, there are two types of design domains i.e., ground structure [22-25] and continuum structures [26-29].

Ground structure uses an exhaustive set of truss or beam/frame elements in the design domain. The individual cross-section is defined as design variables. When the cross sectional area of an element goes to zero, that element will be removed. Thus after the optimization procedure converges, some elements will be removed from the original exhaustive set. The remaining elements will define the topology for the compliant mechanism $[30,20]$. Figure 4 shows examples of initial guess in 2 and 3 dimensions, which is full ground structure with a uniform distribution of cross sectional areas.
In the continuum structures, design domain is typically divided into appropriate finite elements where every element has intrinsic structural properties [31]. In solving topology optimization problems using this kind of domain (continuum), three major approaches are used. One is the homogenization method, which is based on the assumption of microstructure in which the properties are homogenized [26, 27, \& 32]. There are three design variables associated with each finite element. Two of them represent the dimensions of the rectangular hole in the element and the last one is for the orientation of the hole as illustrated in Figure 5. The element is considered anisotropic due to the hole.

Another approach is the density method which the material density of each element is selected as the design variables. The density method

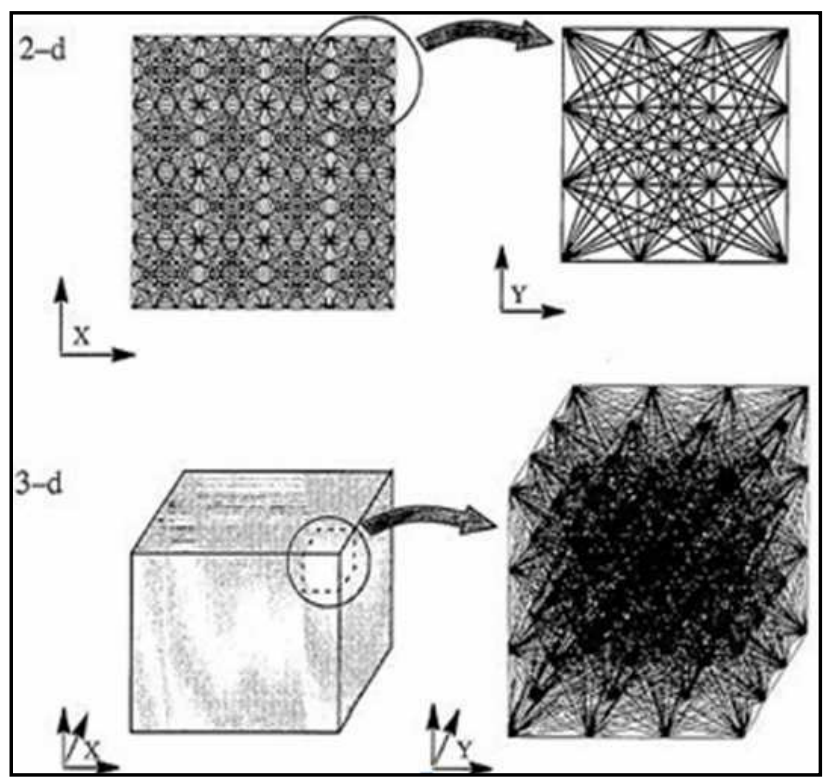

Figure 4: Composite materials composed of truss or thin frame modeled microstructures in 2 and 3 dimensions (Sigmund O. 1995)

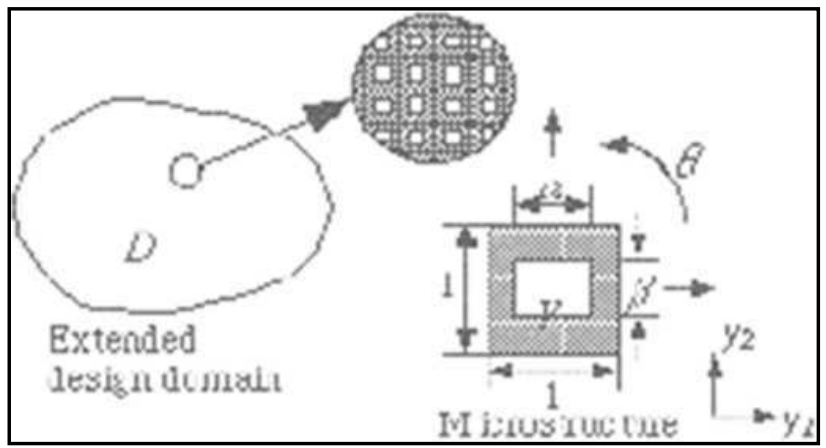

Figure 5: Design parameterization in homogenization method (Li, Y., K. Saito and N.Kikuchi, 1999) 
assumes the material to be isotropic and each design variables varies between zero and one and the intermediate values should be penalized to obtain a "black and white" (zero-one) design $[33,34]$. Several penalization techniques have been suggested. In the SIMP approach (Solid Isotropic Microstructure with Penalization), a power law model is used, where intermediate densities give very little stiffness in comparison to the amount of material used. Another approach is to add a concave penalty function that suppresses intermediate values to the objective function [35, 36].

The third approach is the evolutionary structural optimisation (ESO). The original idea of this method is to gradually remove lowly stressed elements to achieve the optimal design $[37,38]$.

\section{THE PSEUDO-RIGID BODY MODEL}

The pseudo-rigid-body model is used to simplify the analysis and design of compliant mechanisms. This method of modeling allows wellknown rigid-body analysis methods to be used in the analysis of compliant mechanisms [39-41] approximated flexible couplers as a rigid link with a length five-sixths of the flexible segment.

It is analyzed compliant mechanisms with small-length flexural pivots. Since the lengths of the flexural members are small relative to the lengths of the rigid segments, the flexural pivots are modeled as kinematic joints at the center of the flexible segment. Torsional springs are used to represent the member stiffness.

The accuracy of this method decreases as the relative length of the flexural member increases, and a different approach is required for compliant mechanisms containing longer flexural pivots [43].

The pseudo-rigid body model (PRBM) $[17,01]$ is used to model the deflection of flexible members using rigid body components that have equivalent force-deflection characteristics. Rigid link mechanisms theory may then be used to analyze the compliant mechanism. Different types of compliant segments require different pseudorigid models that predict the deflection path and force-deflection relationship of a flexible segment. Figure 6 depicts the pseudo-rigid body model of a large deflection beam, in which it has been assumed that the almost circular path can be accurately modeled by two rigid links joined at a pivot along the beam [01]. This model opened the wealth of information available in rigid body mechanisms synthesis to be used in compliant mechanisms design. The approach is useful for designing mechanisms to perform a traditional task of kinematic synthesis path following, function generation and rigid-body guidance without concern for the energy storage in the flexible members. This flexure based compliant mechanisms can be divided in two main categories: planar (two dimensional) and spatial (three dimensional), depending on the design and overall motion of the mechanism.

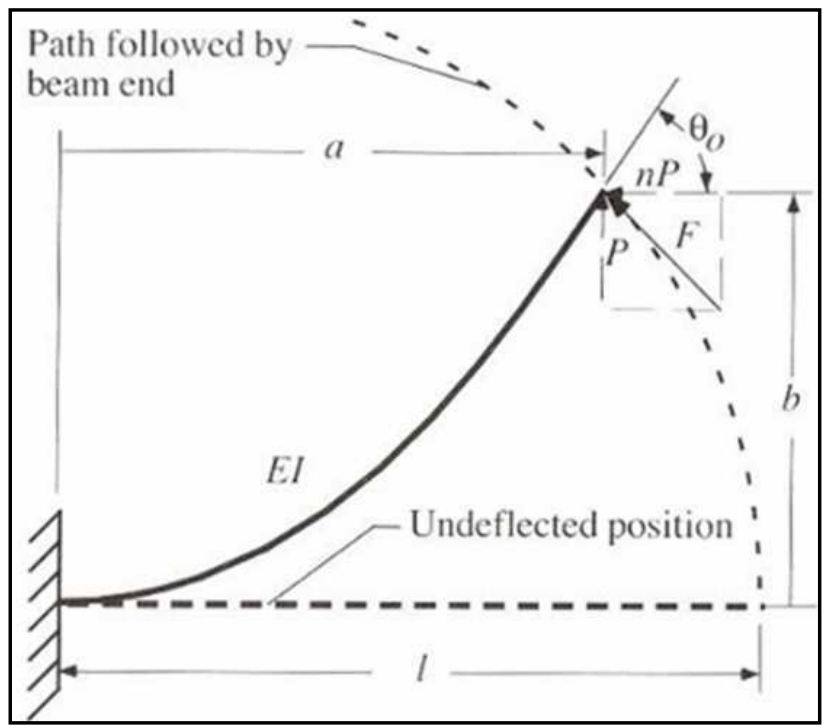

(a)

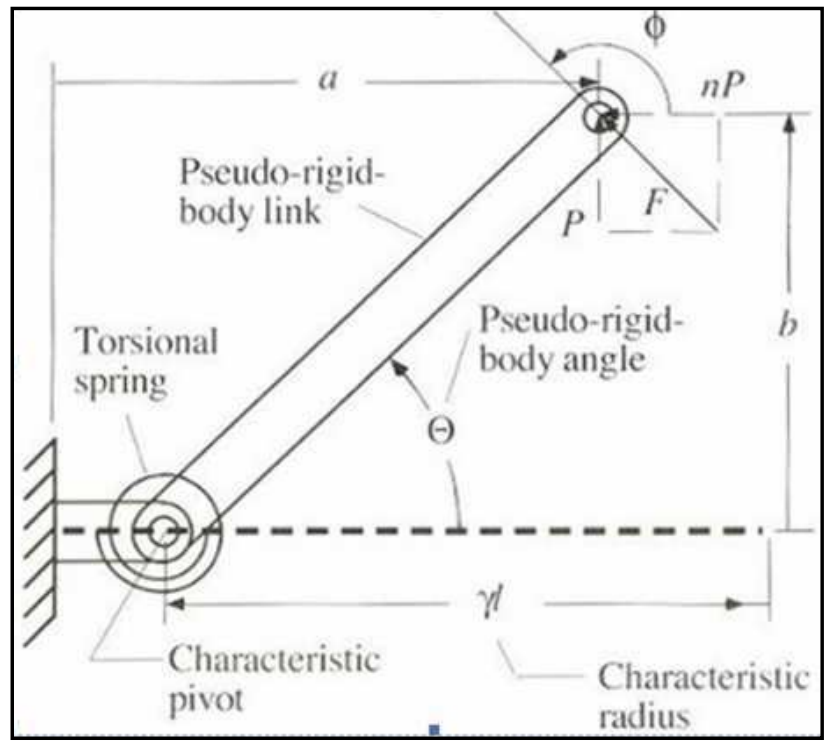

(b)

Figure 6: Large-deflection beam (a) and its pseudorigid body model (b) [01] 


\section{METHODS OF OPTIMIZATION}

There are number of programming techniques may be used in order to solve the problem in structural optimization based approach. For an example, the sequential linear programming (SLP) method is the most popular approximations method for non-linear optimization problem due to its simplicity [22]. However, there are other sophisticated optimization methods such as sequential quadratic programming (SQP), convex linearization (CONLIN), method of moving asymptotes (MMA), generalized convex approximation (GCA), Topology Optimization Method and others. The SLP starts with trial design and replaces the objective function and constraints by linear approximations obtained from a Taylor series expansion about this initial design. An easy-to-use evolutionary structural optimization (ESO) has also been used for solving large structural optimization problem [01].

SQP is a feasible direction method in which the first step is to generate a search direction by solving a sub-problem with quadratic objective functions and linear restrictions, and the algorithm tries to improve the design in such direction (references). The search direction and the objective function are both expanded using Lagrange multipliers, and an exterior penalty is used to free the one-dimensional search from restrictions $[43,44]$.

GMMA is an asymptotic optimization algorithm in which the objective functions and the restrictions can be treated separately because each function has its own moving asymptote. By changing the asymptotic parameters a new family of convex approximation is generated, and this property adds robustness to the method. As the sign of the first derivative remains unaltered trough the optimization (even with the variation of design variables), this algorithm has a monotonic behavior, [45].

GCM derives from GMMA. It's a second order method and as such it needs certain information from a previous iteration (the fist iteration is always of order one). Unlike GMMA, the approximation of GCM is a not monotonic function, and it's suitable for problems where the objective function has a non-linear response to the variation of the design variables, [45].

\section{Topology Optimization Method}

The goal of the Topology Optimization is to de- termine the best use of material for a body using Optimality criteria Method such that the objective criteria take out a maximum or minimum value subject to constraints (Mass or volume Reduction).In topological optimization, the function approach employs mechanical advantage, geometrical advantage and work ratio as objective functions, while displacement constraint and material constraint are imposed to narrow the domain of feasible search. In topological optimization, the material distribution function over a body serves as optimization parameter. The user needs to define the structural problem (material properties, FE model, loads, etc.) and the objective function (i.e., the function to be minimized or maximized) and the state variables (i.e., constrained dependent variables) must be selected among a set of predefined criteria. Midha (2005) discussed, the theory of topological optimization seeks to minimize or maximize the objective function (f), subject to the constraints (gj) defined. The design variables (ni) are internal.

\section{Design of Compliant Mechanism with Point Constraints}

Figure 7 shows the basic design domain. The initial topology specification is as follows, V0 is the maximum possible volume of material of the domain taken for the analysis during the topology optimization process. The input location (xi), input force $(\mathrm{Fi})$, Output displacement $(\delta 0)$ at the output location ( $\mathrm{XO}), \mathrm{X}$ and $\mathrm{Y}$ are the reference axis and $\mathrm{xm}$ is the maximum length of the domain.

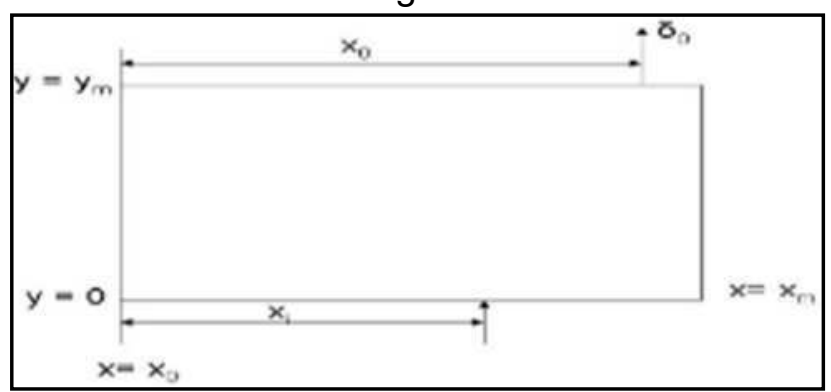

Figure 7: Basic design domain

While doing the analysis, the factors that are kept constant are size of the rectangular domain, boundary conditions (all corners of the design domain are fixed), input force and output location (X0). To study the effect of input location, the input force $(\mathrm{Fi})$ has been applied at various input locations $(\mathrm{Xi})$ of the domain. The input location $\mathrm{Xi}=\mathrm{Xi}, / \mathrm{Xm}$ is varied. 
Figure 8 shows the basic design domain with boundary conditions and applied force, when the force is applied at Xi. Xm is the maximum length of the domain. The output displacement is obtained in $\mathrm{y}$ direction at $\mathrm{X} 0$.

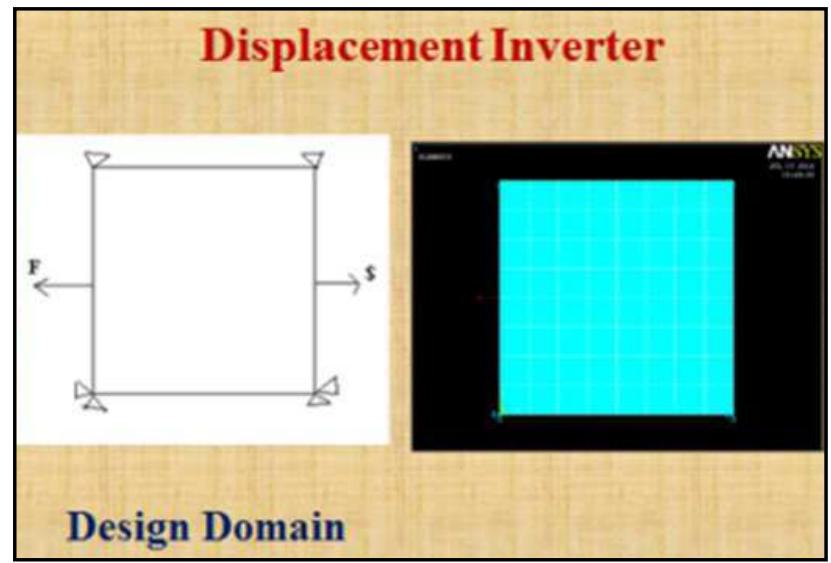

Figure 8: The basic Design domain with boundary conditions \& applied force

The topology optimization has been carried out for the design domain. The topology has converged after 90 iterations. The converged plot is shown in Figure 9.The material distribution is high at the red colour region. The material distribution (density) values are near zero in the blue colour regions as shown in the Figure 10.

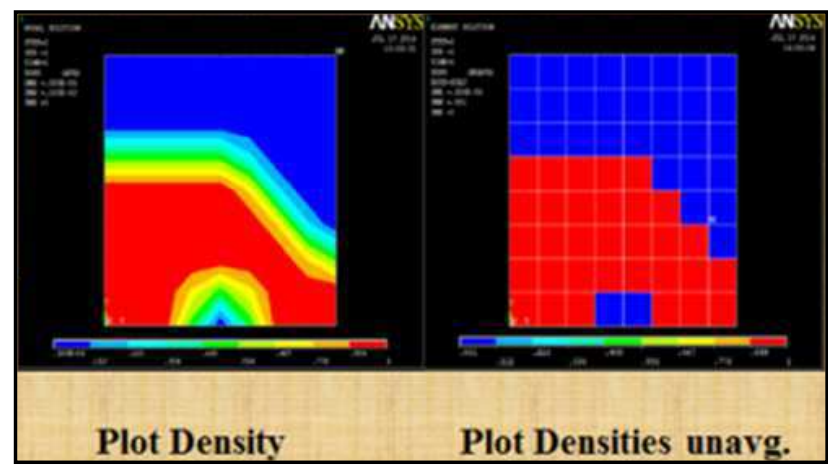

Figure 9 and 10: Density plot - The element density in a topological problem ranges 0 to 1(where the material is not required)

Figure 11 shows the converged solution after topological optimized design. The effect of output location (X0) can be defined as the ratio of length of output location from $x$-axis to the maximum length of the domain. The maximum output displacement value $(\delta 0)$ obtained at $(\mathrm{x} 0 / \mathrm{xm})$.

\section{CONCLUSION}

Compliant mechanisms have made an huge contribution in the design process of various fields such as automotive industry, aerospace industry,
MEMS, Medical devices, Robotic arm with minimal impedance due to its own mass \& Assistive mechanisms, for adaptive structures, components in transportations, hand-held tools, etc. A brief review of the most utilized compliant mechanisms offer many inherent benefits including low cost, zero backlash, ease of manufacture, scalability and in reducing the number of components which therefore Reduces manufacturing cost and additionally increase the performance.

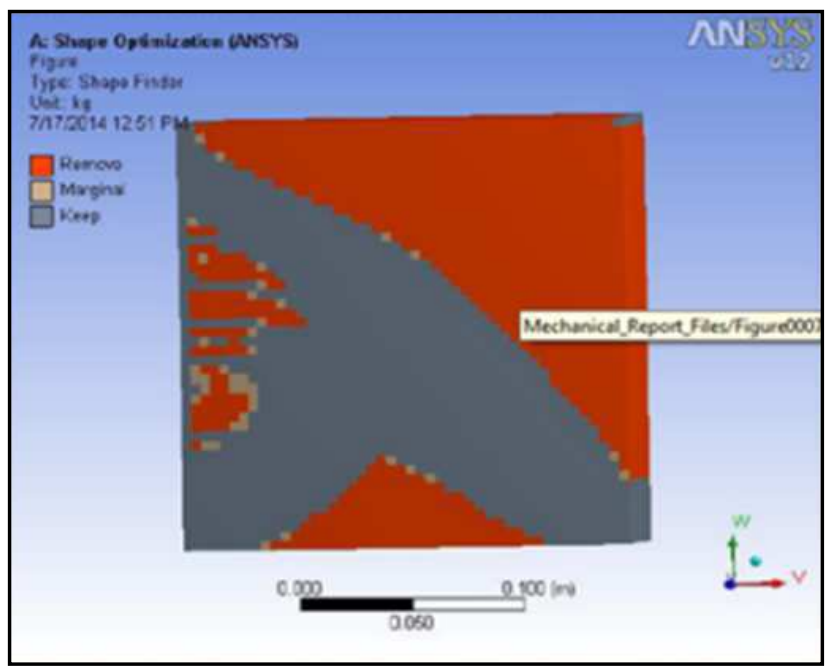

Figure 11: The converged solution after topological optimized domain

Structural optimization techniques are more general design methods, in which a multitude of possible designs are obtained without the need of any commitments or initial topological proposals. However, they are expensive method compared to pseudorigid-body-model and inverse analysis model.

Therefore, lots of researches have been agreed to overcome these problems by using and introducing numerous techniques.

At present work is being carried out to create a basic design domain, initial topology specification, maximum possible volume of the material of the domain and to study the effect of input location, constraints and output. After this to analyze, which method of optimization is feasible for designing a compliant mechanisms.

In general, solution algorithms, Optimality Criteria (OC) method have been used for topology optimization. The inputs required for topology optimization are size and shape of the basic design domain, location of physical supports, the location and direction of the applied force from the displacement inverter, and a limit on the total material resource or volume. In addition, the location and direction of the desired amplifier output are to be specified. 
Optimality Criteria (OC) methods are well suited for problems with a small number of constraints compared to the number of design variables and are generally more computationally efficient than mathematical programming methods. Since there is only one active constraint (the material resource constraint), the OC method can be used to provide more rapid convergence compared to the SQP algorithm, SLP algorithm and Method of Moving asymptotes (MMA).

\section{REFERENCES}

1) Howell, L.L. (Eds.), 2001. Compliant Mechanisms. John Wiley \& Sons, Inc. United States of America.

2) L. L. Howell and A. Midha. A method for the design of compliant mechanisms with smalllength flexural pivots. ASME Journal of Mechanical Design, 115:280-290, 1994.

3) M. D. Murphy, A. Midha, and L. L. Howell. The topological synthesis of compliant mechanisms. Mechanism and Machine Theory, 31:185-199, 1996.

4) G. K. Ananthasuresh. PhD Thesis. A New Design Paradigm for Micro-Electro-Mechanical Systems and Investigations on the Compliant Mechanism Synthesis. University Of Michigan, Ann Harbor, USA, 1994.

5) Canfield, S. and M. Fracker, 2000. 'Topology Optimization of Compliant Mechanical Amplifiers', Journal of Structural Multidisc Optimization, 20: 269-279.

6) Anandhasuresh G.K. and A. Saxena, 2000. 'On an Optimal Property of Compliant Topologies', Journal of Structural Multidisc Optimization, 19: 36-49.

7) Wang M.Y., S. Chen and Y. Mei, 2005. 'Design of Multimaterial Compliant Mechanisms using Level-set Methods', Journal of Mechanical Design, 127: 941-956.

8) Ananthasuresh, G. K., Kota, S., and Kikuchi, K., 1994, "Strategies for Systematic Synthesis of Compliant MEMS," 1994 Int. Mechanical Engineering Congress and Exposition, ASME, Chicago, IL, 55, pp. 677-686.

9) Frecker, M. I., Ananthasuresh, G. K., Nishiwaki, S., Kikuchi, N., and Kota, S., 1997, "Topological Synthesis of Compliant Mechanisms Using Multi-Criteria Optimization," J. Mech. Des., 119_2_, pp. 238-245.

10) Hetrick, J., and Kota, S., 1999, "An Energy
Formulation for Parametric Size and Shape Optimization of Compliant Mechanisms," J. Mech. Des., 121, pp. 229-234.

11) Joo, J., Kota, S., and Kikuchi, N., 2000, "Topological Synthesis of Compliant Mechanisms Using Linear Beam Elements," Mech. Based Des. Struct. ach., 28_4_, pp. 245-280.

12) Mortensen, C.R., B.L. Weight, L.L. Howell and S.P. Magleby, 2000. Compliant mechanism prototyping. In: Proc. of DETC'00, ASME 2000 Design Engineering Technical Conferences \& Computers \& Information in Engineering Conference Baltimore, Maryland.

13) Larsen, U.D., O. Sigmund and S. Bouwstra, 1997. Design and fabrication of compliant micromechanisms and structures with negative poisson's ratio. J. Microelectromech. Syst., 6: 99- 106.

14) Chen, L., 2001. Microfabrication of heterogeneous, optimized compliant mechanisms. NSF Summer Undergraduate Fellowship in Sensor Technologies (SUNFEST 2001), University of Rochester, Advisor, G.K. Ananthasuresh.

15) Sigmund, O., 1997. On the design of compliant mechanisms using topology optimization. Mech. Struct. \& Machines, 25: 493-524.

16) Solehuddin, S., A. Jamaluddin, M. Abd.Samad and A.M. Nizam, 2002. A novel design of a compliant one-piece nail clipper. In: Proc. of the 2nd World Engineering Congress, 2002 WEC.

17) Howell, L.L. and A. Midha, 1996. A loop-closure theory for the analysis and synthesis of compliant mechanisms. SME J. Mech. Design, 118: 121- 125.

18) Ananthasuresh, G.K., S. Kota and Y. Gianchandani, 1994. A methodical approach to the synthesis of micro-compliant mechanisms. Tech. Digest, Solid-State Sensor and Actuator Workshop (held on Hilton Head Island, SC), pp: 189-192.

19) Saxena, A. and G.K. Ananthasuresh, 1998. An optimality criteria approach for the topology synthesis of compliant mechanisms. In: Proc. Of DETC'98, ASME Design, Engineering Technical Conf. (held in Atlanta, GA), DETC'98/MECH5937.

20) Saxena, A. and G.K. Ananthasuresh, 2000. On an optimal property of compliant topolo- 
gies. Struct. Multidisc. Optim., 19: 36-49.

21) Sigmund, O., 2000. Topology optimisation: A tool for the tailoring of structures and materials. Phil. Trans. R. Soc. Lond. A, 358: 211227.

22) Frecker, M.I., 1997. Optimal design of compliant mechanisms. Ph. D. Thesis, Mechanical Engineering in the University of Michigan.

23) Canfield, S. and M. Frecker, 2000. Topology optimisation of compliant mechanical amplifiers for piezoelectric actuators. Struct. Multidisc. Optim., 20: 269-279.

24) Pedersen, C.B.W., 2002. Topology optimisation of energy absorbing frames. In: Proc. of WCCM V, 5th World Congress on Computational Mechanics.

25) Ansola, R., J. Canales, J.A. Tarrago and J. Rasmussen, 2002. On simultaneous shape and material layout optimisation of shell structures. Struct. Multidisc. Optim., 24: 175184.

26) Li, Y., K. Saito and N. Kikuchi, 2002. Design of heat-actuated compliant mechanism and its application to product-embedded disassembly. In: Proc. 5th World Congress on Computational Mechanics (WCCM V), Vienna, Austria.

27) Nishiwaki, S., M.I. Frecker, S. Min and N. Kikuchi, 1998. Topology optimisation of compliant mechanisms using the homogenization method. Int. J. Numer. Meth. Engng., 42: 535-559.

28) Hammer, V.B. and N. Olhoff, 2000. Topology optimisation of continuum structures subjected to pressure loading. Struct. Multidisc. Optim., 19: 85- 92.

29) Pedersen, C.B.W., T. Buhl and O. Sigmund, 2001. Topology synthesis of large-displacement compliant mechanisms. Int. J. Numer. Meth. Engng., 50: 2683-2705.

30) Frecker, M., S. Kota and N. Kikuchi, 1999. Topology optimisation of compliant mechanisms with multiple outputs. Struct. Optim., 17: 269-278.

31) Fuchs, M.B. and E. Moses, 2000. Optimal structural topologies with transmissible loads. Struct. Multidisc. Optim., 19: 263-273.

32) Allaire, G. and C. Castro, 2002. Optimisation of nuclear fuel reloading by the homogenisation. Struct. Multidisc. Optim., 24: 11-22.
33) Chen, T.Y. and C.Y. Lin, 2000. Determination of optimum design spaces for topology optimization. Finite Elements in Analysis and Design, 36: 1-16.

34) Borrvall, T. and J. Petersson, 2001. Topology optimisation using relularized intermediate density control. Comput.. Methods Appl. Mech. Engrg., 190: 4911-4928.Stolpe, M. and Svanberg, K. 2001, "On the Trajectories of Penalization Methods for Topology Optimisation", Struct. Multidisc Optim Vol. 21, pp. 128-139.

35) Stolpe, M. and K. Svanberg, 2001. An alternative interpolation scheme for minimum compliance topology optimization. Struct. Multidisc. Optim., 22: 116-124.

36) Hsu, Y.L., M.S. Hsu and C.T. Chen, 2001. Interpreting results from topology optimization using density contours. Computers \& Structures, 79: 1049-1058.

37) Bulman, S., J. Sienz and E. Hinton, 2001. Comparisons between algorithms for structural topology optimisation using a series of benchmark studies. Computers \& Structures, 79: 1203-1218.

38) Salman, B.A., 1989, "Mechanical Advantage Aspects in Compliant Mechanisms Design," M.S. thesis, Purdue University, West Lafayette, IN.

39) Burns, R.H., 1964, "The Kinetostatic Synthesis of Flexible Link Mechanisms," Ph.D. dissertation, Yale University, New Haven. CT.

40) Burns, R.H., and Crossley, F.R.E., 1968, "Kinetostatics Synthesis of Flexible Link Mechanisms," ASME Paper 68-Mech-5.

41) Howell, L.I.., and Midha, A., 1993, "Compliant Mechanisms," Section 9.10 in Modern Kinematics: Developments in the last Forty Years, (A.G. Erdman, ed), Wiley, New York, pp. 422-428.

42) K. Schittkowski. Optimization in industrial en gineering: Sqp - methods and applications. RadiossUser Meeting, Mecalog, Nice, 2005.

43) Belegundu, A. and T. Chadruparla, 1999. 'Optimization Concepts and Applications in Engineering', Upper Saddle River, New Jersey, Prentice Hall Publications.

44)A. Remouchamps and Y. Radovcic. Boss quattro: an open system for parametric design. JournalStructural and Multidisciplinary Optimization, 23:140-152, 2002. 
45) (46.) Deepak, R., M. Dinesh, D.K. Sahu and G.K. Ananthasuresh, 2009. 'A Comparative Study of the Formulations and Benchmark Problems for the Topology Optimization of Compliant Mechanisms', Journal of Mechanisms and Robotics, 1: 215-222.

46) (47.) Frecker, M. and S. Kota, 1997. 'Topological Synthesis of Compliant Mechanisms Using Multi-Criteria Optimization', Journal of Mechanical Design, Transaction of ASME, 119: 238-245.

47) (48.) Kikuchi, N. and S. Nishiwaki, 1998. 'Design Optimization method for Compliant Mechanisms and Material Microstructure', Journal of Computer Methods in Applied Mechanics and Engineering, 151: 401-417.
48) (49.) Bendsoe, M.P. and N. Kikuchi, 1998. 'Generating Optimal Topologies in Structural Design Using a Homogenization Method', Journal of Computer Methods in Applied Mechanics and Engineering, 71: 197-224.

49) (50.) Hetric, J.A. and S. Kota, 1999. 'An Energy Formulation for Parametric Size and Shape Optimization of Compliant Mechanisms', ASME Journal of Mechanical Design, 121: 229-234.

50) (51.) K. J. Lu and S. Kota. An effective method of synthesizing compliant adaptive structures using load path representation. Journal of Intelligent Systems and Structures, 16:307-317, 2005.

Paper sent to revision: 06.05.2015.

Paper ready for publication: 18.04.2016. 